\title{
Trend of Cryptosporidium Infection among Children below 24 Months in an Informal Urban Settlement, Kenya
}

\author{
Daisy Chepkemoi Mutai' ${ }^{1}$, Patrick Opiyo Owili', Miriam Adoyo Muga² \\ ${ }^{1}$ Department of Public Health, School of Health Sciences, University of Eastern Africa, Baraton, Kenya \\ ${ }^{2}$ Department of Human Nutrition and Dietetics, School of Medicine and Health Sciences, Kabarak University, Nakuru, Kenya \\ Email: daisy.mutai@yahoo.com, owilip@ueab.ac.ke, mmuga@kabarak.ac.ke
}

How to cite this paper: Mutai, D.C., Owili, P.O. and Muga, M.A. (2020) Trend of Cryptosporidium Infection among Children below 24 Months in an Informal Urban Settlement, Kenya. Open Journal of Medical Microbiology, 10, 153-161.

https://doi.org/10.4236/ojmm.2020.103013

Received: July 28, 2020

Accepted: September 6, 2020

Published: September 9, 2020

Copyright $\odot 2020$ by author(s) and Scientific Research Publishing Inc. This work is licensed under the Creative Commons Attribution International License (CC BY 4.0).

http://creativecommons.org/licenses/by/4.0/

\begin{abstract}
Cryptosporidium infection is estimated to cause 2.9 million diarrheal cases yearly among children aged under 24 months in sub-Saharan Africa. Studies have shown long-term climatic variations can affect infectious diseases. The burden of cryptosporidiosis in rural areas of sub-Saharan Africa is well characterized. However, the trend of Cryptosporidium infection is not known, especially in informal urban settings. This study therefore sought to determine cryptosporidiosis trends, and further explore the association between year and Cryptosporidium infection among children below 24 months in Kibera urban informal settlement in Kenya. Data collected by the Kenya Medical Research Institute longitudinal study in Tabitha clinic in Kibera from 2009 to 2015 were used. At least 3000 children aged $<24$ months receive free health care at the clinic. In the longitudinal study, children presenting with diarrhea were eligible for stool sample collection $(n=477)$, out of which 421 stool samples were tested using TaqMan ${ }^{\mathrm{TM}}$ Array Card (TAC) polymerase chain reaction panel that included a target for Cryptosporidium genus. Data for the 421 children were included in the analysis. Logistic regression was used to explore the difference between the seven years and cryptosporidiosis. Overall, the pooled data indicated that $23.5 \%$ of the children who were tested had Cryptosporidium infection, with the highest proportions of Cryptosporidium-positive cases observed in 2015 (45.2\%). The logistic regression results also indicated that children who were tested in the year 2015 were more likely to have Cryptosporidium infection ( $\mathrm{OR}=3.39 ; 95 \% \mathrm{CI}: 1.44-7.96 ; p=0.005)$ than those in 2009. Watery stool was also found to be an important symptom of cryptosporidiosis. There was a high prevalence of Cryptosporidium infection among young children, especially in the most recent year. Routine testing of Cryptosporidium infection using molecular methods, constant moni-
\end{abstract}


toring and identification of the infection sources is therefore necessary towards reducing the disease burden in the low resource settings.

\section{Keywords}

Cryptosporidium Infection, Informal Urban Settlement, Children below 24 Months, Trend, TaqMan ${ }^{\mathrm{TM}}$ Array Card

\section{Introduction}

Cryptosporidium is a protozoan parasite which is a common cause of gastrointestinal illnesses in children [1]. The current global statistics suggest that the burden of Cryptosporidium infection is between 15\% - 25\% among children with diarrhea [2]. This has shown a significant increase from earlier studies that were reported in a systematic review indicating a prevalence of $1 \%$ among children in high-income countries and 5\% - 10\% in low- and middle-income countries [3]. This increase could be as result of several factors including the environmental and behavioral change over time.

The clinical manifestations of Cryptosporidium infection in children are associated with their immunity status that may include profuse, non-bloody watery diarrhea that usually resolves within 48 hours. The severity of diarrheal illness could be exhibited by a diarrheal duration of 7 to 14 days or more in addition to symptoms such as fever, abdominal cramps, vomiting, lethargy, and general malaise [1] [4].

In sub-Saharan Africa, cryptosporidiosis is estimated to occur in 2.9 million children below two years annually, which also increases the mortality rate among children aged between 12 to 23 months [2]. Some studies in Africa have revealed that the prevalence of Cryptosporidium infection among children was $12.3 \%, 10 \%$ and 9\% in Ethiopia, Botswana and Kenya, respectively [4] [5] [6]. However, most of these studies did not consider the trend of Cryptosporidium infection over time. Moreover, these positive cases were found to occur majorly during diarrheal outbreak periods and among children who were less than 5 years.

The World Health Organization (WHO), in 2004 integrated cryptosporidiosis into the neglected tropical diseases due to its occurrence among the children in low resourced settings, where inaccessibility to services, inadequate resources and harsh weather patterns are the main drivers [7]. In low resourced settings, particularly where cases and risk of deaths due to cryptosporidiosis are high, immunocompromised individuals and under-5 children are disproportionately affected with no antiprotozoal drug or vaccine for effective disease management currently existing [8].

One study in Kenya found that $46 \%$ of under- 5 children who were Cryptosporidium positive were living in semi-urban areas [9]. This high prevalence of Cryptosporidium infection in semi-urban areas is worrying since Kenya has the 
largest informal urban settlement in Africa, that is, Kibera slums. To develop public health preventive strategies, an understanding of the trend and the current situation was therefore necessary, and this informed the basis for conducting our study in an informal urban settlement in Kenya. Kibera slum being the largest slum in Africa increases the population at risk of Cryptosporidium infection due to several environmental hygiene factors such as lack of access to safe water for drinking and poor sanitation facilities [6] [10]. Therefore, this study was aimed to determine the trend of cryptosporidiosis, and further explore the association between the change in time (i.e. years) and Cryptosporidium infection among children below 24 months in Kibera informal urban settlement in Kenya.

\section{Methods}

\subsection{Study Design and Data Source}

A longitudinal data collected by Kenya Medical Research Institute (KEMRI) in Kibera's Gatwikira and Soweto villages among children were used. KEMRI in collaboration with Tabitha clinic offered free health care for the study participants who were under surveillance for several acute illnesses. The clinic and laboratory test results for children aged 24 months and below who were presented with acute diarrhea at Tabitha Clinic from 2009 to 2015 were used.

\subsection{Sampling and Laboratory Processing}

A convenience sampling technique was employed in the selection of children to be included in the study. A total of 22,660 children who were below 24 months (female $=11,265$; male $=11,395)$ were identified as having visited the clinic from the year 2009 to 2015. Children who presented with symptoms of loose stools and had stool samples collected $(n=477)$ were then selected from the dataset. Out of the 477, those who were eligible for this study were only 421 whose samples were tested for cryptosporidiosis using enteric $\mathrm{TaqMan}^{\mathrm{TM}}$ Array Card (TAC) real-time polymerase chain reaction (PCR) following the protocol described by Liu and colleagues [11].

\subsection{Data Management and Analysis}

Data containing children information including demographic, symptoms and laboratory test results for Cryptosporidium infection, were extracted from the database and saved in Microsoft Excel format before cleaning, checking for discrepancies and analyses. Descriptive statistics were used to determine the prevalence of Cryptosporidium infection by year, age, gender, and clinical characteristics in the study. The differences between categories of the variables were tested using Pearson's Chi-square test while logistic regression modeling technique was used to explore the association between the change in time (year) and Cryptosporidium infection among children below 24 months. Both the descriptive and inferential statistics were presented in the form of tables. All the analyses were 
conducted using STATA version 13.0.

\subsection{Ethical Consideration}

Ethical approval was sought from the institutional ethics committee of the University of Eastern Africa, Baraton (UEAB) (UEAB/REC/03/10/2019) and the National Commission for Science, Technology and innovation (NACOSTI) (NACOSTI/P/19/2689). Permission to use the dataset was granted by KEMRI. For this study, there were no risks to the study participants since all the data were anonymized.

\section{Findings}

The analysis of the pooled data of 421 children indicated that close to a quarter of them had Cryptosporidium infection (23.5\%) with those tested in the year 2015 presenting the highest proportion (45.2\%) of infection (Table 1). Also, nearly a third of children who had watery stool (29.6\%) had Cryptosporidium infection while a quarter of those who were vomiting tested positive for cryptosporidiosis. Moreover, $27.0 \%$ of the children who had stopped breastfeeding tested positive for Cryptosporidium infection, however the difference between the groups was not statistically significant.

The odds ratios (ORs) and 95\% confidence intervals (CI) of the association between year and Cryptosporidium infection among children below 24 months in Kibera urban informal settlement in Kenya are presented in Table 2. The year was positively associated with cryptosporidiosis before and after adjustment. After adjusting for all the variables, a positive trend was exhibited as from the year 2013 to 2015 with the increased odds of $1.29,1.42$ and 3.39, respectively, though the years 2013 and 2014 were not statistically significant. However, the children who were tested in the year 2015 were more likely to have cryptosporidiosis (OR $=3.39 ; 95 \%$ CI: $1.44-7.96)$ than their counterparts who were tested in the year 2009. Watery stools remained a key symptom of Cryptosporidium infection with children who had watery stool being 3.04 times more likely to be infected (95\% CI: 1.74 - 5.28). The children who were vomiting were however less likely to have Cryptosporidium infection but was marginally significant. Age, gender and presenting with fever were not significantly associated with cryptosporidiosis.

\section{Discussion}

This study was carried out in the largest informal urban settlement in Africa (i.e., Kibera slums) to explore the trend of Cryptosporidium infection among children aged 24 months and below. We found that $45.2 \%$ of the children were Cryptosporidium positive in 2015-a higher prevalence detected using real-time molecular PCR method. Our results support the findings of a recent study which found a $46 \%$ prevalence of Cryptosporidium infection among under-5 children who were living in semi-urban areas [9]. The pooled data for the seven years however revealed a $23.5 \%$ prevalence. The prevalence found in this study was 
higher than what had been previously reported in other studies carried out in urban (4\%) and rural (9\%) areas among the children below 24 months in Kenya [1] [9]. Other studies conducted in Africa have also reported a much lower prevalence $($ Egypt $=17 \%$ and Ethiopia $=12 \%)$ of Cryptosporidium infection among the under-five than what was found in this study [4] [12].

Table 1. Descriptive statistics of children aged $0-24$ months, by Cryptosporidium infection.

\begin{tabular}{|c|c|c|c|c|}
\hline \multirow{2}{*}{ Variables } & \multicolumn{4}{|c|}{ Cryptosporidium lab results } \\
\hline & Total $n$ & Negative, $n(\%)$ & Positive, $n(\%)$ & $P$-value ${ }^{\mathrm{a}}$ \\
\hline Total number & 421 & $322(76.5)$ & $99(23.5)$ & \\
\hline Year & & & & 0.015 \\
\hline 2009 & 79 & $60(76.0)$ & $19(24.1)$ & \\
\hline 2010 & 92 & $79(85.9)$ & $13(14.1)$ & \\
\hline 2011 & 58 & $45(77.6)$ & $13(22.4)$ & \\
\hline 2012 & 36 & $28(77.8)$ & $8(22.2)$ & \\
\hline 2013 & 43 & $32(74.4)$ & $11(25.6)$ & \\
\hline 2014 & 71 & $55(77.5)$ & $16(22.5)$ & \\
\hline 2015 & 42 & $23(54.8)$ & $19(45.2)$ & \\
\hline Age group & & & & 0.612 \\
\hline$\leq 12$ months & 176 & $132(75.0)$ & $44(25.0)$ & \\
\hline 13 - 18 months & 140 & $106(75.7)$ & $34(24.3)$ & \\
\hline 19 - 24 months & 105 & $84(80.0)$ & $21(20.0)$ & \\
\hline Gender & & & & 0.197 \\
\hline Female & 211 & $167(79.2)$ & $44(20.8)$ & \\
\hline Male & 210 & $155(73.8)$ & $55(26.2)$ & \\
\hline Breastfeeding & & & & 0.598 \\
\hline No & 37 & $27(73.0)$ & $10(27.0)$ & \\
\hline Yes & 384 & $295(76.8)$ & $89(23.2)$ & \\
\hline \multicolumn{5}{|l|}{ Clinical presentation } \\
\hline Had watery stool & & & & 0.001 \\
\hline No & 191 & $160(83.8)$ & $31(16.2)$ & \\
\hline Yes & 230 & $162(70.4)$ & $68(29.6)$ & \\
\hline Had fever $\left(\geq 38.0^{\circ} \mathrm{C}\right)$ & & & & 0.763 \\
\hline No & 375 & $286(76.3)$ & $89(23.7)$ & \\
\hline Yes & 46 & $36(78.3)$ & $10(21.7)$ & \\
\hline Had vomit & & & & 0.076 \\
\hline No & 316 & $235(74.4)$ & $81(25.6)$ & \\
\hline Yes & 105 & 87 (82.9) & $18(17.1)$ & \\
\hline
\end{tabular}

a, $P$-value was obtained using Pearson Chi-Square to test the statistical difference between the categories. 
Table 2. Logistic regression results of the Cryptosporidium infection of children aged 0 - 24 months.

\begin{tabular}{|c|c|c|}
\hline \multirow{2}{*}{ Variables } & Crude Model & Adjusted Model \\
\hline & OR $(95 \% \mathrm{CI})$ & OR (95\% CI) \\
\hline \multicolumn{3}{|l|}{ Year } \\
\hline 2009 & 1 & 1 \\
\hline 2010 & $0.52(0.24,1.14)$ & $0.52(0.23,1.16)$ \\
\hline 2011 & $0.91(0.41,2.04)$ & $0.94(0.41,2.17)$ \\
\hline 2012 & $0.90(0.35,2.31)$ & $0.98(0.37,2.61)$ \\
\hline 2013 & $1.09(0.46,2.56)$ & $1.29(0.52,3.21)$ \\
\hline 2014 & $0.92(0.43,1.96)$ & $1.42(0.62,3.29)$ \\
\hline 2015 & $2.61(1.18,5.79)^{\star *}$ & $3.39(1.44,7.96)^{\star * *}$ \\
\hline \multicolumn{3}{|l|}{ Age group } \\
\hline$\leq 12$ months & 1 & 1 \\
\hline $13-18$ months & $0.96(0.57,1.61)$ & $0.99(0.58,1.72)$ \\
\hline $19-24$ months & $0.75(0.42,1.35)$ & $0.75(0.40,1.40)$ \\
\hline \multicolumn{3}{|l|}{ Gender } \\
\hline Female & 1 & 1 \\
\hline Male & $1.35(0.86,2.12)$ & $1.49(0.92,2.42)$ \\
\hline \multicolumn{3}{|l|}{ Still breastfeeding } \\
\hline No & 1 & 1 \\
\hline Yes & $0.81(0.38,1.75)$ & $0.66(0.30,1.48)$ \\
\hline \multicolumn{3}{|c|}{ Clinical presentation } \\
\hline \multicolumn{3}{|l|}{ Had watery stool } \\
\hline No & 1 & 1 \\
\hline Yes & $2.17(1.34,3.49)^{\star \star \star}$ & $3.04(1.74,5.28)^{\star * * *}$ \\
\hline \multicolumn{3}{|l|}{ Had fever, $\geq 38.0^{\circ} \mathrm{C}$} \\
\hline No & 1 & 1 \\
\hline Yes & $0.89(0.43,1.87)$ & $0.95(0.43,2.11)$ \\
\hline \multicolumn{3}{|l|}{ Had vomit } \\
\hline No & 1 & 1 \\
\hline Yes & $0.60(0.34,1.06)^{*}$ & $0.55(0.30,1.01)^{*}$ \\
\hline
\end{tabular}

OR, Odds ratio; CI, Confidence interval; ${ }^{\star} p \leq 0.10 ;{ }^{* *} p \leq 0.05 ;{ }^{* * *} p \leq 0.01 ;{ }^{* * *} p \leq 0.001$.

Nonetheless, most of these studies in Africa used various conventional diagnostic techniques for detection of Cryptosporidium infection which are less sensitive compared to molecular diagnostic test that was employed in our study. This would therefore explain a much higher prevalence in this study than the rest that were conducted in other parts of Africa. Clearly, this would mean that 
there has been an underreporting of the Cryptosporidium infections in Africa because of the diagnostic techniques that have been used before. Hence, it is necessary that future studies should consider using real-time molecular PCR method rather than the conventional diagnostic techniques. In addition, the different study design methods and study settings in the studies carried out in Africa could also explain the differences in prevalence that were found.

Furthermore, it is necessary to establish a surveillance system for Cryptosporidium infection within the country among other infectious diseases. Our study has revealed that there was an association between the change in time (year) and rate of cryptosporidiosis among children, with children who were born in the most recent years being more likely to be affected. Several public health intervention strategies are therefore necessary since the rates of infections are expected to be higher now than before. The reasons for this association and high infections in the recent years may be due to environmental degradation and low hygienic conditions in the informal urban settlement brought about by increased population over the years. Therefore, exerting more pressure on the existing water and sanitation facilities in the area.

This study also found that children who were presented with watery stool were 3.04 times more likely to test positive for Cryptosporidium infection. This association was also observed in a study in Pakistan [13]. Even though, this study found that children who were presented with vomiting were less likely to test positive, it remained marginally significant after adjustment. Other studies, however, found a positive association between vomiting and cryptosporidiosis [9] [12]. The lack of distinguishable clinical signs or symptoms of cryptosporidiosis in Kibera slums suggests that a detailed examination and screening for this infection should be performed especially in endemic areas [14].

This study had limitations and strengths. First, we used a secondary data that had the limitation of important variables such as the sources and treatment of drinking water, food handling and preparation practices as well as environmental and socioeconomic conditions. Therefore, we were unable to adjust for several important potential confounders. Secondly, this study could not establish whether the symptoms presented could have been as a result of other diarrheal infecting pathogens that are usually common among these children. Hence, the findings of this study should be interpreted with a lot of caution. However, some of the strengths in this study included the use of real-time molecular PCR method to diagnose cryptosporidiosis unlike other studies that used conventional diagnostic techniques. This gave accurate results of the infection. The other strength is also in relation to data that were collected over the seven-year period. We were able to explore the change in infection over time.

\section{Conclusion}

In this study, we found a positive trend in the Cryptosporidium infection among children below 24 months in Kibera urban informal settlement, with the preva- 
lence being $45.2 \%$ in 2015. Moreover, we found an increase in the rate of infections in the most recent years. Routine testing of Cryptosporidium infection using molecular methods, constant monitoring and identification of the infection sources should be considered. This would necessitate the development and implementation of public health policies on health promotion and preventive strategies that will facilitate the reduction of the disease burden among the low-income populations. Future studies should therefore endeavor to explore the seasonality as well as the sources of cryptosporidiosis among children in Kibera.

\section{Acknowledgements}

The authors are appreciative to Kenya Medical Research Institute (KEMRI) and its collaborators for allowing us to use their data for this study.

\section{Conflicts of Interest}

The authors declare no conflicts of interest regarding the publication of this paper.

\section{References}

[1] Delahoy, M.J., et al. (2018) Clinical, Environmental, and Behavioral Characteristics Associated with Cryptosporidium Infection among Children with Moderate-to-Severe Diarrhea in Rural Western Kenya, 2008-2012: The Global Enteric Multicenter Study (GEMS). PLOS Neglected Tropical Diseases, 12, e0006640. https://doi.org/10.1371/journal.pntd.0006640

[2] Sow, S.O., et al. (2016) The Burden of Cryptosporidium Diarrheal Disease among Children < 24 Months of Age in Moderate/High Mortality Regions of Sub-Saharan Africa and South Asia, Utilizing Data from the Global Enteric Multicenter Study (GEMS). PLoS Neglected Tropical Diseases, 10, e0004729. https://doi.org/10.1371/journal.pntd.0004729

[3] Dillingham, R.A., Lima, A.A. and Guerrant, R.L. (2002) Cryptosporidiosis: Epidemiology and Impact. Microbes and Infection, 4, 1059-1066.

https://doi.org/10.1016/S1286-4579(02)01630-1

[4] Wegayehu, T., Adamu, H. and Petros, B. (2013) Prevalence of Giardia duodenalis and Cryptosporidium Species Infections among Children and Cattle in North Shewa Zone, Ethiopia. BMC Infectious Diseases, 13, 419.

https://doi.org/10.1186/1471-2334-13-419

[5] Anne Alexander, K., Herbein, J. and Zajac, A. (2012) The Occurrence of Cryptosporidium and Giardia Infections among Patients Reporting Diarrheal Disease in Chobe District, Botswana. Advances in Infectious Diseases, 2, 143-147. https://doi.org/10.4236/aid.2012.24023

[6] Kotloff, K.L., et al. (2013) Burden and Aetiology of Diarrhoeal Disease in Infants and Young Children in Developing Countries (the Global Enteric Multicenter Study, GEMS): A Prospective, Case-Control Study. The Lancet, 382, 209-222.

[7] Savioli, L., Smith, H. and Thompson, A. (2006) Giardia and Cryptosporidium Join the "Neglected Diseases Initiative". Trends in Parasitology, 22, 203-208. https://doi.org/10.1016/j.pt.2006.02.015 
[8] Sponseller, J.K., Griffiths, J.K. and Tzipori, S. (2014) The Evolution of Respiratory Cryptosporidiosis: Evidence for Transmission by Inhalation. Clinical Microbiology Reviews, 27, 575-586. https://doi.org/10.1128/CMR.00115-13

[9] Mbae, C. (2008) Cryptosporidiosis: Prevalence, Genotype Analysis, and Symptoms Associated with Infections in Children in Kenya. International Journal of Infectious Diseases, 12, e72. https://doi.org/10.1016/j.ijid.2008.05.180

[10] Peletz, R., Mahin, T., Elliott, M., Montgomery, M. and Clasen, T. (2013) Preventing Cryptosporidiosis: The Need for Safe Drinking Water. Bulletin of the World Health Organization, 91, 238-238. https://doi.org/10.2471/BLT.13.119990

[11] Liu, J., et al. (2013) A Laboratory-Developed TaqMan Array Card for Simultaneous Detection of 19 Enteropathogens. Journal of Clinical Microbiology, 51, 472-480. https://doi.org/10.1128/JCM.02658-12

[12] Abdel-Messih, I.A., et al. (2005) Diarrhea Associated with Cryptosporidium parvum among Young Children of the Nile River Delta in Egypt. Journal of Tropical Pediatrics, 51, 154-159. https://doi.org/10.1093/tropej/fmh105

[13] Khan, A., Shams, S., Khan, S., Khan, M.I., Khan, S. and Ali, A. (2019) Evaluation of Prevalence and Risk Factors Associated with Cryptosporidium Infection in Rural Population of District Buner, Pakistan. PLoS ONE, 14, e0209188. https://doi.org/10.1371/journal.pone.0209188

[14] Dabas, A., Shah, D., Bhatnagar, S. and Lodha, R. (2017) Epidemiology of Cryptosporidium in Pediatric Diarrheal Illnesses. Indian Pediatrics, 54, 299-309. https://doi.org/10.1007/s13312-017-1093-3 